\title{
TESTING CONVECTIVE-CORE OVERSHOOTING USING PERIOD SPACINGS OF DIPOLE MODES IN RED GIANTS
}

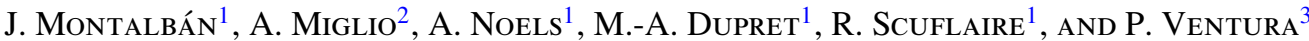 \\ ${ }^{1}$ Institut d'Astrophysique et Géophysique de l'Université de Liège, Allée du six Août, 17 B-4000 Liège, Belgium \\ ${ }^{2}$ School of Physics and Astronomy, University of Birmingham, Edgbaston, Birmingham B15 2TT, UK \\ ${ }^{3}$ Osservatorio Astronomico di Roma-INAF, via Frascati 33, I-00040 Monteporzio Catone, Rome, Italy \\ Received 2012 December 7; accepted 2013 February 13; published 2013 March 15
}

\begin{abstract}
Uncertainties on central mixing in main-sequence (MS) and core He-burning (He-B) phases affect key predictions of stellar evolution such as late evolutionary phases, chemical enrichment, ages, etc. We propose a test of the extension of extra-mixing in two relevant evolutionary phases based on period spacing $(\Delta P)$ of solar-like oscillating giants. From stellar models and their corresponding adiabatic frequencies (respectively, computed with ATON and LOSC codes), we provide the first predictions of the observable $\Delta P$ for stars in the red giant branch and in the red clump (RC). We find (1) a clear correlation between $\Delta P$ and the mass of the helium core $\left(M_{\mathrm{He}}\right)$; the latter in intermediatemass stars depends on the MS overshooting, and hence it can be used to set constraints on extra-mixing during MS when coupled with chemical composition; and (2) a linear dependence of the average value of the asymptotic period spacing $\left(\langle\Delta P\rangle_{a}\right)$ on the size of the convective core during the He-B phase. A first comparison with the inferred asymptotic period spacing for Kepler RC stars also suggests the need for extra-mixing during this phase, as evinced from other observational facts.
\end{abstract}

Key words: asteroseismology - convection - stars: evolution - stars: late-type - stars: low-mass

Online-only material: color figures

\section{INTRODUCTION}

Despite the numerous efforts undertaken to understand convection in stars, the treatment of this process in stellar modeling is still rather simplistic and one of the major uncertainties affecting the predictions of stellar evolution theory. In particular, while convection is a highly non-local phenomenon, the extension of the convective region is determined by local criteria such as Schwarzschild and Ledoux ones. Nowadays, there is clear evidence that the current description of convection is unsatisfactory: numerical simulations, laboratory experiments, and disagreement between theoretical predictions and observations. For instance, mixing beyond the convective core formal limit during the main sequence (MS) is needed to reproduce the morphology of color-magnitude diagrams of stellar clusters and the properties of binary systems (see, e.g., Maeder \& Mermilliod 1981; Andersen et al. 1990; Ribas et al. 2000). Similarly, observational evidence suggests that the extension of the central mixed region during the core He-burning (He-B) phase should be larger than determined by the Schwarzschild criterion. In fact, this extension has important consequences for the duration of the He-B phase, but also in the following evolutionary phases, determining, for instance, the ratio between asymptotic giant branch and horizontal branch stars. Moreover, the different chemical profiles of $\mathrm{C}$ and $\mathrm{O}$ from different kinds of mixing directly affect the oxygen abundance of white dwarfs (see, e.g., Straniero et al. 2003). The nature of the mechanism(s) inducing extra-mixing, both in MS and He-B phases, as well as its extension is still debated (see, e.g., Chiosi 2007 for review).

Stellar seismology tries to answer some of these questions by looking for seismic indices based on different oscillation modes and asymptotic relationships, or deviations with respect to them (see, e.g., Noels et al. 2010 for review). In this context, the most powerful diagnostics are those based on oscillation modes that propagate close to the central region, in particular, gravity modes (g-modes) and mixed gravity-pressure modes. Some of these seismic indices were successfully applied to individual stars showing different kind of pulsations: solar-like oscillations in MS stars and sub-giants (Di Mauro et al. 2003; Miglio \& Montalbán 2005; Deheuvels \& Michel 2011) and Btype pulsators (Aerts et al. 2003; Dziembowski \& Pamyatnykh 2008; Degroote et al. 2010).

Here, we show that solar-like oscillation modes in G-K red giants (RGs) provide a most effective tool to test convectivecore overshooting, both during the MS and the core-He-burning phase. Moreover, thanks to Kepler and CoRoT observations, such tests can potentially be performed on a large number (thousands) of stars encompassing a wide range of stellar parameters.

\section{RED GIANTS: INTERNAL STRUCTURE AND EVOLUTION}

The evolution of post-MS stars is characterized by a contracting $\mathrm{He}$ core and an expanding $\mathrm{H}$-rich envelope, with an $\mathrm{H}$-burning shell in between that becomes thinner and thinner as the star evolves on the red giant branch (RGB). The contracting core releases thermal energy, part of which is used to increase the temperature as long as the gas is not too degenerate, but which no longer produces heating in highly degenerate conditions. For a given He-core mass, there is a maximum temperature that can be reached by core contraction as any further contraction leads to gas cooling. Highly degenerate cores cannot ignite helium burning if their mass is lower than about $0.475 M_{\odot}$. Such stars keep ascending the RGB until their He cores reach this critical value (see, for instance, Kippenhahn \& Weigert 1990; Sweigart et al. 1990).

For total masses lower than about $2 M_{\odot}$, the He-core mass at the onset of the He flash and at the start of the post flash He-B phase $(\mathrm{ZAHeB})$ is thus about the same, i.e., $\sim 0.475 M_{\odot}$. 
With the increase of the total mass, the electron degeneracy level is lower and the He-core mass required for He ignition decreases. After reaching a minimum value, the He-core mass increases again following the total mass, as a result of the larger and larger convective core mass during the MS phase (Girardi et al. 1998; Castellani et al. 2000 and references therein). Stars with the minimum He-core mass $\left(\sim 0.33 M_{\odot}\right.$, the lowest mass for a pure He star to start burning He-see, e.g., Kippenhahn \& Weigert 1990) then define a transition between two different behaviors of RG evolution (Sweigart et al. 1990). Helium-burning stars with masses smaller than the transition stellar mass $\left(M_{\mathrm{tr}}\right)$ populate the so-called red clump (RC; Girardi et al. 1998). Since the luminosity of He-B stars depends mainly on the He-core mass, the luminosity of RC stars is approximately constant, it rapidly decreases near the transition, and then increases drastically as the total mass increases. The stellar mass at which the transition occurs depends on the He-core mass at the end of the MS, and hence on the chemical composition and on the amount of overshooting in the MS models. He-B stars with masses close to the transition mass form the so-called secondary clump (Girardi 1999).

The models presented in this paper were computed with the stellar evolution code ATON (Ventura et al. 2008). We followed the evolution from pre-MS to central He exhaustion of stellar models with masses from 0.7 to $4.0 M_{\odot}$, following the He-flash for low-mass models. Figure 1 (upper panel) shows the $\mathrm{He}$-core mass at He ignition as a function of the stellar mass for models computed without and with core overshooting during the MS evolution. For the chemical composition considered, the transition mass is $2.4 M_{\odot}$, while the value decreases to $2.2 M_{\odot}$ for models with overshooting. A similar decrease is obtained by decreasing the metallicity by a factor of two.

\section{ADIABATIC OSCILLATION PROPERTIES: PERIOD SPACING}

The properties of oscillation modes depend on the behavior of the Brunt-Väisälä $(N)$ and Lamb $\left(S_{\ell}\right)$ frequencies. Because of the high-density contrast between the core and the envelope, the $\mathrm{RG}$ oscillation spectrum is characterized by a large number of mixed p-g modes in addition to the radial ones (see, for instance, Christensen-Dalsgaard 2004; Montalbán et al. 2010 for details).

In RGs, once the temperature for He ignition is reached, convection appears in the nuclear burning core and is accompanied by the expansion of the star central regions. The core structure is therefore radically different from that of an RGB star, characterized by a high-density electron-degenerate radiative helium core (see Figure 2). Both the presence of a convective core and the decrease of the density contrast $\left(\rho_{\mathrm{c}} /\langle\rho\rangle\right)$ determine significant changes in the Brunt-Väisälä frequency distribution near the core, and therefore in the seismic properties of dipole modes (Montalbán et al. 2010). The asymptotic approximation for g-modes (Tassoul 1980) predicts that the periods of two modes of the same degree $(\ell)$ and consecutive order $(n)$ are separated by a constant value $\langle\Delta P\rangle_{\mathrm{a}}$ :

$$
\langle\Delta P\rangle_{\mathrm{a}}=\frac{2 \pi^{2}}{\sqrt{\ell(\ell+1)}} \frac{1}{\int N / r d r} .
$$

In the He-B model, the expansion of the central layers leads to a lower $N$ maximum, and its location is displaced at larger stellar radius. Moreover, the central convective regions do not contribute to the integral in Equation (1) (see Figure 2, upper panel, for a detailed description). The period spacing between

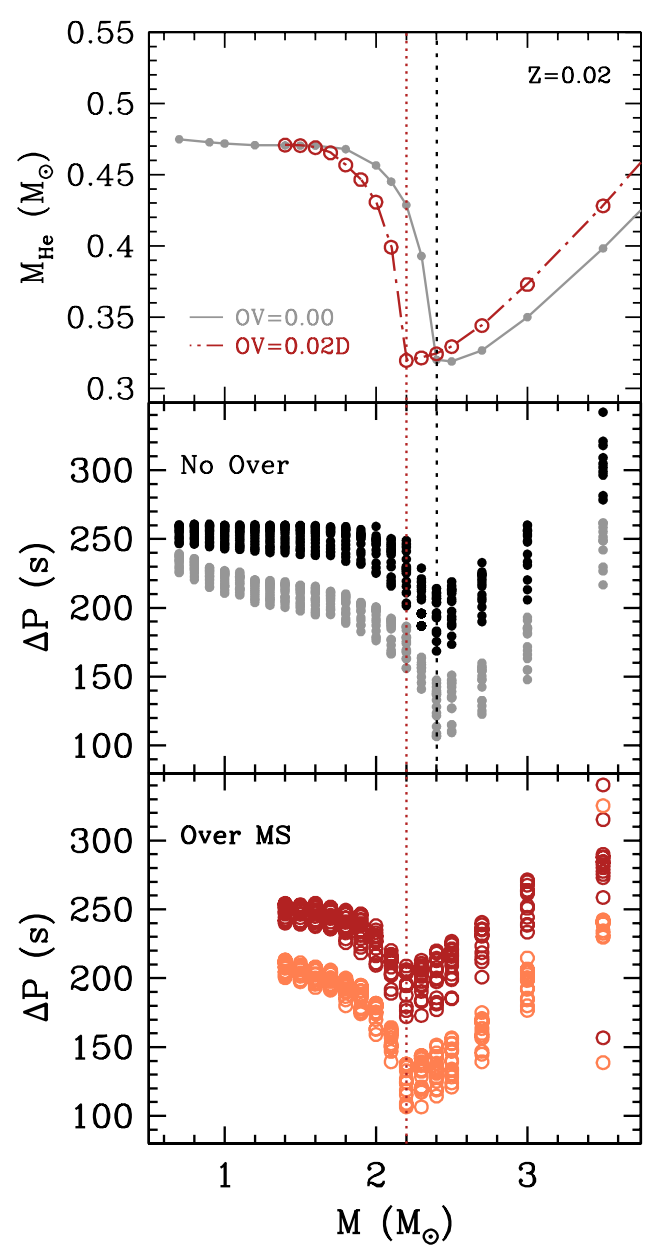

Figure 1. Upper panel: He-core mass at He ignition vs. stellar mass. Solid line and dots correspond to models without overshooting during the MS phase, and dashed line and open circles to models computed with diffusive overshooting (Ventura et al. 1998). Central panel: average period spacing during the He-B phase $\left(0.9>Y_{\mathrm{C}}>0.1\right)$ vs. stellar mass. Black dots correspond to $\langle\Delta P\rangle_{a}$ and gray ones to $\langle\Delta P\rangle_{\text {th-obs. }}$ Lower panel: as central panel for models with overshooting during MS phase. Vertical lines indicate the transition mass for no-overshooting models (small-dashed line) and MS overshooting ones (dotted line).

(A color version of this figure is available in the online journal.)

consecutive g-modes, as determined from Equation (1), is therefore significantly smaller in the RGB model (60 s) than in the He-B one (240 s).

The asymptotic approximation is, however, no longer valid for mixed modes (those observed in RGs). For a detailed comparison with the observations, we therefore compute adiabatic oscillation frequencies using the Eulerian version of the code LOSC (Scuflaire et al. 2008). The results are shown in the lower panels of Figure 2 where we plot the mode inertia of radial and dipole modes, as well as the period separation between dipole modes of consecutive radial order $n(\Delta P=P(n+1)-P(n))$, as a function of the mode frequencies. In addition to a significant difference in the period spacing itself, the differences between the spectra can be summarized as follows: (1) in the RGB model, the inertia of $\ell=1$ pressure-dominated modes (corresponding to local minima in $E$ ) is closer to that of the radial models, indicating a weaker coupling between gravity and acoustic cavities compared to the He-B phase. This is also evident from the gravity-dominated modes of RGB, for which $\Delta P$ is almost constant (as expected for pure g-modes) except 

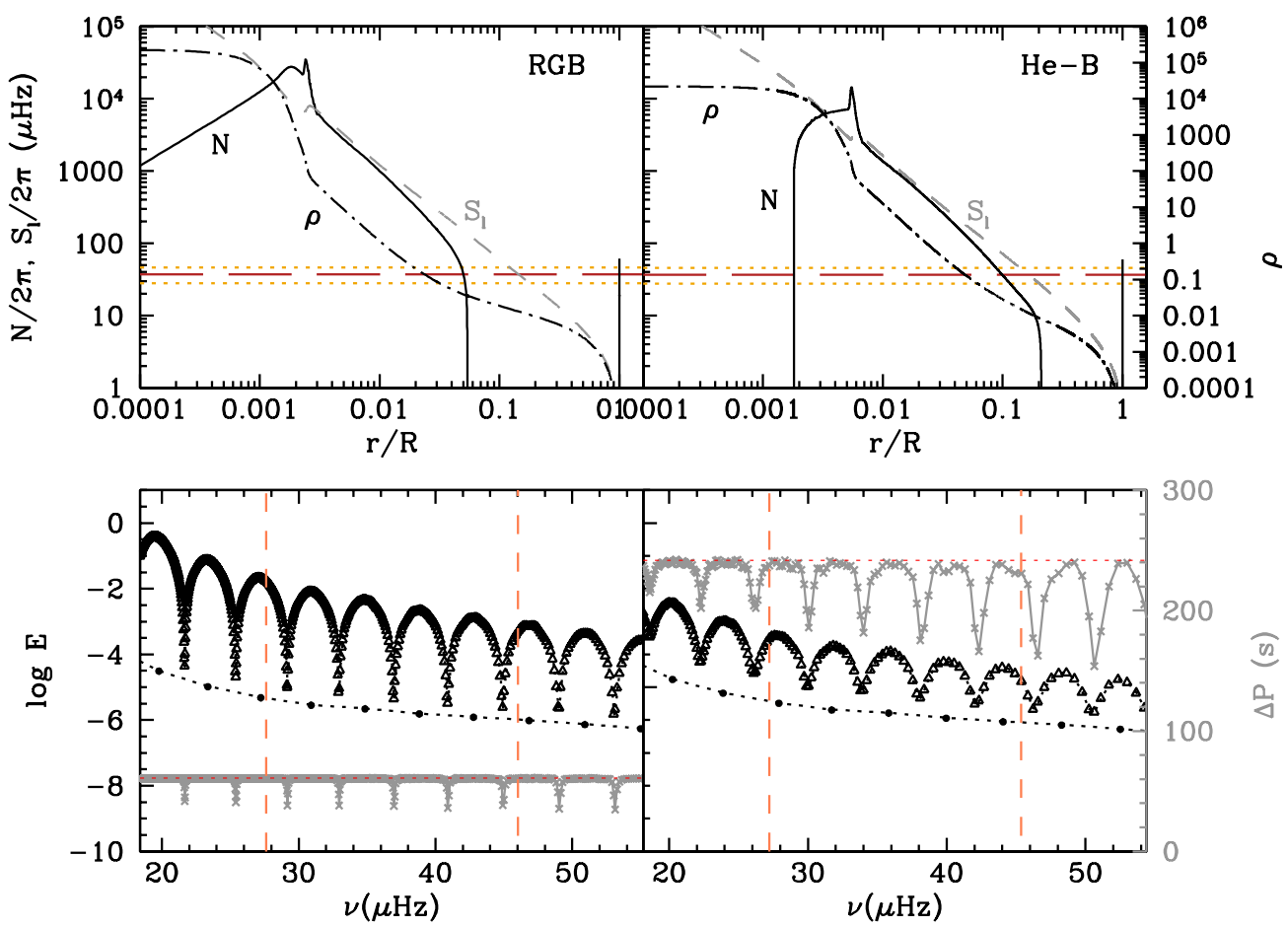

Figure 2. Upper panels: density distribution and $\ell=1$ propagation diagram for a $1.5 M_{\odot}$ star with radius $\sim 12 R_{\odot}$ : in the ascending red giant branch (RGB, left) and in the red clump (He-B, right). Horizontal dotted lines denote the frequency domain of solar-like oscillations. The solid line is the Brunt-Väisälä frequency, and the dashed one the Lamb frequency for $\ell=1$. The dash-dotted line represents density (scaled on right axis). The He-B model has a small convective core $\left(r_{\mathrm{cc}} \simeq 0.002 R\right.$ and $\left.m_{\mathrm{cc}} \simeq 0.08 \mathrm{M}\right)$ and its central density is 10 times smaller than for the RGB one. Due to electron degeneracy, $N$ in the RGB model is significantly lower in the deep central layers than near the H-burning shell. Lower panels: corresponding plots of inertia $(E)$ vs. frequency for the $\ell=0$ (circles) and 1 (triangles) modes. The vertical dashed lines correspond to the horizontal ones in the upper panels. The gray crosses and lines represent period separation between consecutive dipole modes vs. frequency (right axis). The horizontal thin-dashed lines correspond to $\langle\Delta P\rangle_{\mathrm{a}}$.

(A color version of this figure is available in the online journal.)

for the modes describing the minimum of inertia, while for the g-p mixed modes of the He-B model, the deviation of $\Delta P$ from a constant value is more important. (2) The density of the $\ell=1$ modes for the He-B model is lower than for the RGB one (by a factor of three for the models shown in Figure 2).

To compare theoretical predictions and observational results, it is mandatory to define theoretical indices as close as possible to the observational ones. To derive the average period spacing equivalent to that measured in Bedding et al. (2011) and Mosser et al. (2011), we should identify, among all the theoretical modes, those that are most likely to be observed, and thus contribute to the observed period spacing value. That would require non-adiabatic computations, which are very time consuming and unfeasible for the large number of models considered in this study. Moreover, from a theoretical point of view, the detectability of mixed modes not only depends on the models (stellar structure and time-dependent convection), which are subject to uncertainties, but it also strongly depends on the duration of observations (Dupret et al. 2009) and to a lesser extent on the instrument. Therefore, following an adiabatic approach, we consider the modes with lower inertia as those most likely to be detected. These are also the modes contributing to significant deviations from the uniform $\Delta P$ predicted by the asymptotic approximation (minima in $\Delta P$, see Figure 2, lower panels). We define $\langle\Delta P\rangle_{\text {th-obs }}$, a theoretical estimation of the measurable period spacing from observed oscillation spectra, following the same procedure as in Bedding et al. (2011) and based on the properties of observed oscillation spectra. We select oscillation modes with angular degree $\ell=0$ and 1 and with frequencies $v$ in the expected solar-like domain, defined as $0.75<v / \nu_{\max }<$
1.25 , where $v_{\max }=M\left(M_{\odot}\right) R\left(R_{\odot}\right)^{-2}\left(T_{\text {eff }} / 5777\right)^{-0.5} 3050 \mu \mathrm{Hz}$. Around each pressure-dominated dipole mode, we consider the values $\Delta P(n)$ involving $k$ modes with frequencies lower and higher than that of the inertia minimum. Our choice $(k=2)$ is based on current observations (three or four values for each radial order). The $\Delta P(n)$ 's obtained in this way are then averaged in the solar-like frequency domain to obtain $\langle\Delta P\rangle_{\text {th-obs. }}$.

The behavior of $\langle\Delta P\rangle_{\text {th-obs }}$ as a function of the average large frequency separation is shown in Figure 3 for models in the RGB and in the central He-B phase with different chemical composition. These results are in good agreement with the recent observational results obtained with Kepler and CoRoT, where the clear difference between the period spacing of the RGB and He-B models allowed us to use $\langle\Delta P\rangle$ to identify the evolutionary state of RGs with comparable $\langle\Delta v\rangle$ or $v_{\max }$ (Bedding et al. 2011; Mosser et al. 2011).

\section{PERIOD SPACING IN THE RED-CLUMP AND SECONDARY-CLUMP STARS: A PROXY FOR THE He-CORE MASS}

Both the predicted and observed (see Bedding et al. 2011; Mosser et al. 2011) period spacings show significant scatter in He-B stars. This dispersion is partly due to the different masses of the stars, chemical compositions, and central helium mass fraction $\left(Y_{\mathrm{C}}\right)$. Mosser et al. (2011) identified the high- $\Delta v-$ low$\Delta P$ tail as corresponding to the secondary clump, and we will show here that asymptotic and "measurable" values of the period spacing contain additional information about the structure and previous evolution of the star. 


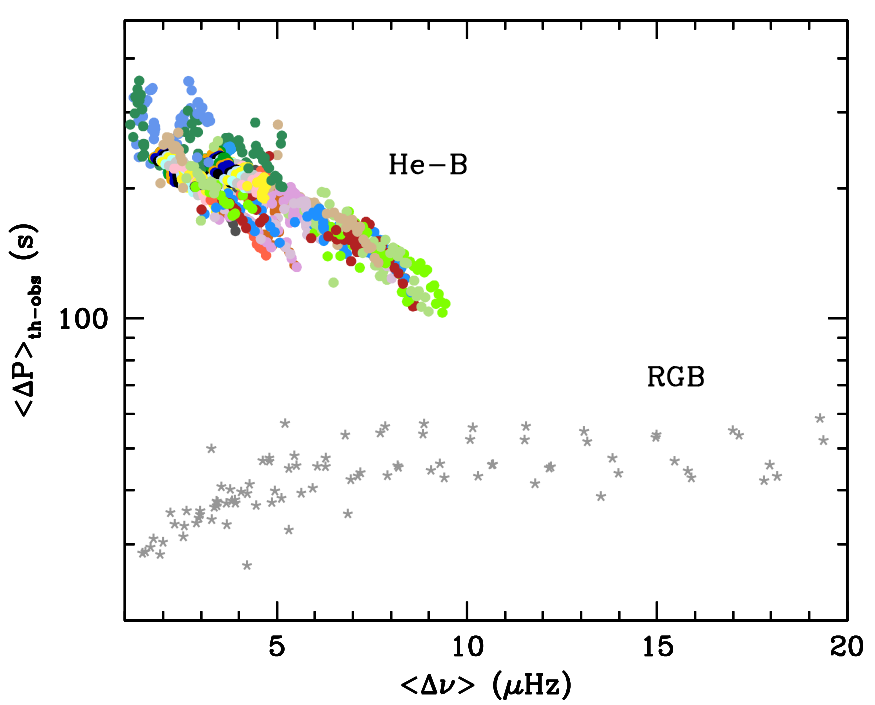

Figure 3. Theoretical "observable" period spacing vs. large frequency separation of radial modes. Asterisks: RGB models with masses 0.9, $1.01 .5,1.6$, and $1.7 M_{\odot}$ and chemical composition $(Z=0.02, Y=0.278)$. Solid dots: He-B models with masses between 0.7 and $4.0 M_{\odot}$ and chemical compositions $(Z, Y)$ : $(0.02,0.278) ;(0.01,0.278) ;(0.02,0.25) ;(0.02,0.4)$. Each color corresponds to models of different stellar masses.

(A color version of this figure is available in the online journal.)

$\langle\Delta P\rangle_{a}$ depends mainly on the value of the $\rho_{c} /\langle\rho\rangle$ (density contrast), on the dimension of the convective core, and on the location of the H-shell which gives rise to a local maximum of $N$. All these quantities change during the He-B phase and they do it differently depending on the stellar mass. In Figure 1 (black dots in central panel), we plot $\langle\Delta P\rangle_{a}$ for models with masses between 0.7 and $3.5 M_{\odot}$ during the He-B phase as a function of the stellar mass. By comparison with the corresponding curve of the upper panel, it seems evident that there is a direct correlation between $\langle\Delta P\rangle_{a}$ and the mass of the He-core. $\langle\Delta P\rangle_{a}$ is almost constant for low-mass stars that begin to burn He in a degenerate core of $0.475 M_{\odot}$ and presents a minimum at the transition mass, which also corresponds to the minimum of the He-mass core $\left(\sim 0.33 M_{\odot}\right)$. For higher masses, $\langle\Delta P\rangle_{a}$ increases with the stellar mass, such as the mass of the He-core $\left(\sim 0.1 M_{T}\right.$ at the end of MS).

$\langle\Delta P\rangle_{\text {th-obs }}$, as described in the previous section, involves additional information related to the coupling between acoustic and gravity cavities, and hence depends on the properties of the evanescent region which can be characterized, roughly speaking, by the value of the integral $\int(\sigma c)^{-1}\left(\left(\sigma^{2}-N^{2}\right)\left(S_{\ell}-\sigma^{2}\right)\right)^{1 / 2} d r$ in the region between the He-core and the envelope. The gray dots in Figure 1 (central panel) represent the corresponding values of $\langle\Delta P\rangle_{\text {th-obs }}$ for models during the He-B phase. The difference between asymptotic and "observable" values also depends on the stellar mass for low-mass models. That should not be surprising since the central density of models with $0.7 M_{\odot}$ and $1.5 M_{\odot}$, their He-core mass, and total radius are almost the same, which leads to a very different density (and temperature) distributions in their envelope, and hence to a very different coupling. Nevertheless, there is still a clear dependence of $\langle\Delta P\rangle_{\text {th-obs }}$ on the He-core mass.

$\langle\Delta P\rangle_{\text {th-obs }}$, like $\langle\Delta P\rangle_{\mathrm{a}}$, presents a minimum corresponding to $M_{\mathrm{tr}}$, whose value strongly depends on the extension of the mixed central region during the MS. In Figure 4, we plot the "observable" period spacing, for models with and without overshooting during the MS, as a function of the He-core mass.

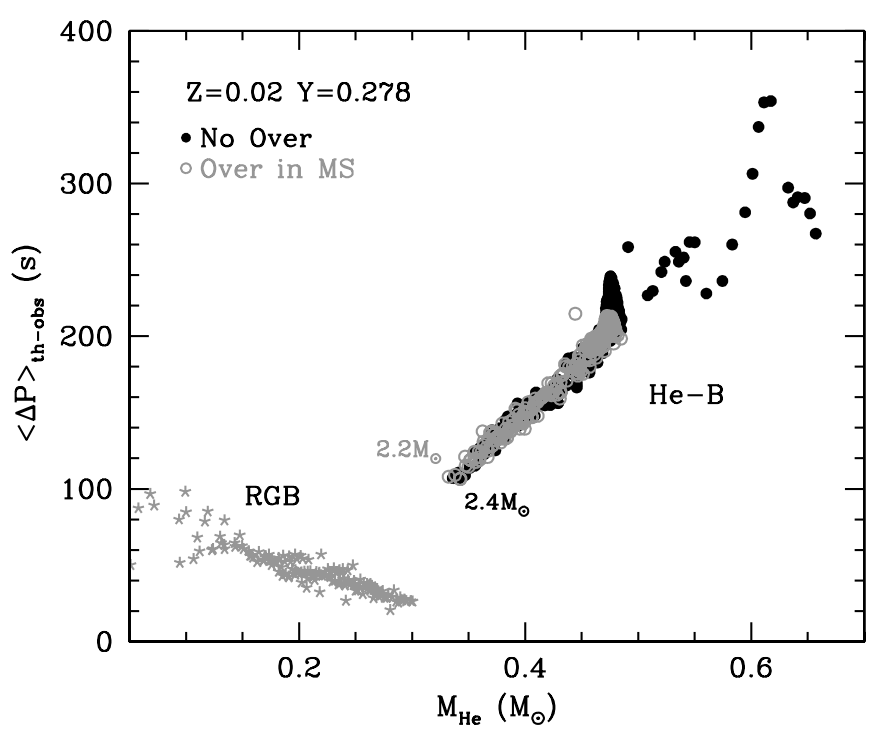

Figure 4. Theoretical "observable" period spacing vs. He-core mass for RGB models (gray asterisks). Black dots: He-B $\left(0.9 \geqslant Y_{\mathrm{C}} \geqslant 0.1\right)$ models with masses between 0.7 and $4.0 M_{\odot}$ without overshooting. Open gray circles: the same for MS-overshooting models and stellar mass between 1.4 and $3.0 M_{\odot}$.

Two linear relationships appear between $\langle\Delta P\rangle_{\text {th-obs }}$ and $M_{\mathrm{He}}$ : the first for low-mass RGB models with $\Delta P$ decreasing as $M_{\mathrm{He}}$ increases due to the larger contrast density as the star evolves, the second one for He-B models. For the latter, both families of models follow the same relation, with the only difference being the mass of the models occupying the same location in that diagram.

In the framework of adiabatic calculations, that is, assuming the amplitude of modes directly linked to $\sqrt{E}$, we use the ratio between the inertia of a mixed mode and that of the corresponding pressure-dominated one to flag that mode as detectable, and thus contributing to the average period spacing value. That ratio depends on details of the stellar structure, and therefore on stellar parameters such as mass, age, and evolutionary state. In fact, we should expect to observe less mixed modes as the inertia ratio increases, which is the case for models with better trapped dipole modes. We are aware that this detectability limit is an arbitrary choice since the relation between inertia and amplitude is only a proxy, nevertheless, the current available non-adiabatic computations confirm that assumption (Dupret et al. 2009; Grosjean et al. 2013). Moreover, we can expect more modes to be detected in longer time series, changing the value of $\Delta P$ obtained from the mean value of individual measured period spacings, and with $\langle\Delta P\rangle_{a}$ being an upper limit.

To see how our results depend on the choice of the detectability limit and on the number of mixed modes considered as observables, we estimated $\langle\Delta P\rangle_{\text {th-obs }}$ while changing these parameters. In Figure 5, we compare the results obtained for He-B models, adopting $k=2$ and a limit value of the inertia ratio (i.e., the standard procedure used in this work in Figures 1, 3, and 4) with the $\langle\Delta P\rangle_{\text {th-obs }}$ values that results when four mixed modes at lower and higher frequency of each pressure-dominated mode in the solar-like frequency domain are considered, and that without imposing any detectability limit. An additional increase of $k$ does not introduce noticeable changes to the behavior of $\langle\Delta P\rangle_{\text {th-obs }}$. Note that $\langle\Delta P\rangle_{\text {th-obs }}$ for lowest mass models is only slightly affected, and that the more important effect is obtained for secondary clump models. It is worth mentioning that in the case of secondary clump stars, such a large number of detected mixed 


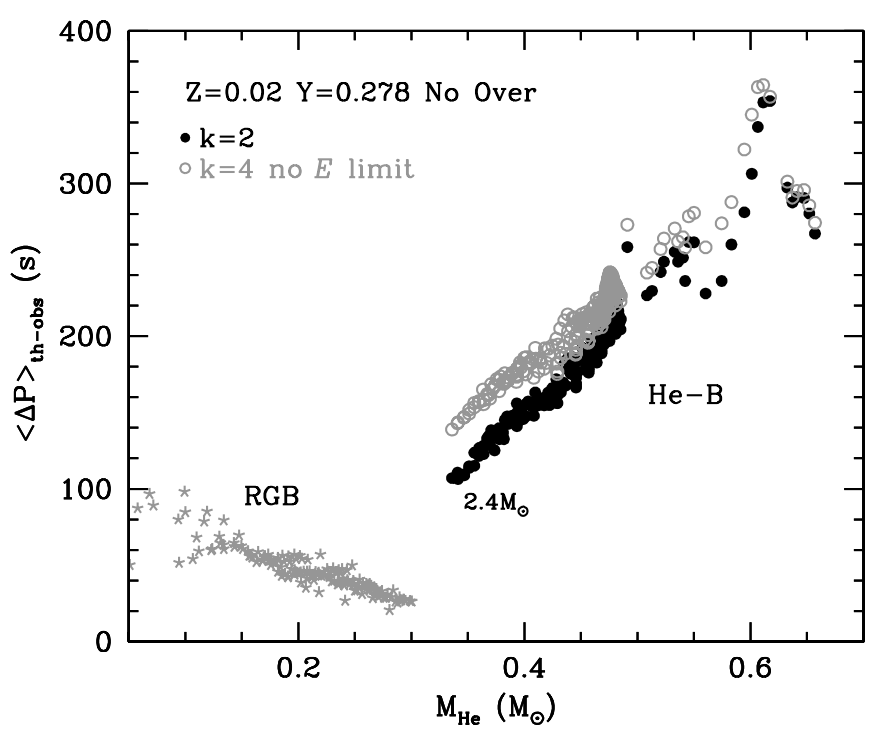

Figure 5. Theoretical "observable" period spacing vs. He-core mass for models computed without overshooting. Black dots: $\langle\Delta P\rangle_{\text {th-obs }}$ computed with our standard method (see the text); gray open circles: $\langle\Delta P\rangle_{\text {th-obs }}$ computed taking four mixed modes at lower and higher frequencies than that of the pressuredominated modes, and without imposing any detectability limit.

modes around the pressure-dominated ones allow us to derive $\Delta P$ as a function of frequency, and then to do a detailed analysis of their stellar structure.

We stress that for a theoretical interpretation of the observations, it is mandatory to use definitions of theoretical and observational indices that are as close as possible. That is true for $\langle\Delta P\rangle_{\text {th-obs }}$, but also for $\langle\Delta v\rangle$ or $\langle\delta v\rangle$. Even if the specific values of $\langle\Delta P\rangle_{\text {th-obs }}$ may change with the number of mixed modes considered, a linear relation between $\langle\Delta P\rangle_{\text {th-obs }}$ and He-core mass remains. The $k$ value must be adapted to the current observations, i.e., changing according to the duration of time series and instrument features.

Period spacing in He-B red giant stars provides a stringent test of the central mixing during the H-MS. However, to successfully exploit this possibility, spectroscopic constraints on the chemical composition are required, together with an accurate estimate of stellar mass: seismic constraints (other than period spacing) will be crucial in this respect.

\section{PERIOD SPACING AS A TEST OF OVERSHOOTING AND MIXING DURING THE CORE-HELIUM BURNING PHASE}

The value of the asymptotic period spacing depends on the central distribution of $N$, and hence on the size of the convective core. Figure 6 shows $\langle\Delta P\rangle_{\mathrm{a}}$ as a function of the convective-core radius $\left(R_{\mathrm{cc}}\right)$ for models in the He-B phase, with $Y_{\mathrm{C}}$ between 0.9 and 0.1 , and masses from 0.7 to $4.0 M_{\odot}$. As can be seen from Figure 6, the relation between $\langle\Delta P\rangle_{\mathrm{a}}$ and $R_{\mathrm{cc}}$ is, to a good approximation, linear.

For a given mass, the trend of $\langle\Delta P\rangle_{\mathrm{a}}$ changes when $Y_{\mathrm{C}} \simeq$ 0.3 due to the much lower temperature dependence of the nuclear reaction ${ }^{12} \mathrm{C}(\alpha, \gamma){ }^{16} \mathrm{O}$ which, at that stage, becomes the dominant energy source in the core. For massive stars, $R_{\mathrm{cc}}$ and $\langle\Delta P\rangle_{\mathrm{a}}$ decrease for models with $Y_{\mathrm{C}}<0.3$ because $\rho_{\mathrm{c}}$ increases as $\mathrm{He}$ is exhausted. For models with $Y_{\mathrm{C}} \gtrsim 0.3$, the mass and radius of the convective core follow a linear relation, which, however, disappears when ${ }^{12} C(\alpha, \gamma){ }^{16} O$ takes over the $3 \alpha$ reaction: in that case, $M_{\mathrm{cc}}$ increases even more quickly but $R_{\text {cc }}$ decreases.

Recently, Christensen-Dalsgaard (2012) and Mosser et al. (2012) showed that $\langle\Delta P\rangle_{a}$ can be inferred from the observed period spacing of dipole mixed modes. Checking the procedure with our theoretical frequencies for $1.5 M_{\odot}$ models of the He-B phase, we were able to recover the asymptotic period spacing with a precision of $2 \%$ when we take into consideration 6-8 mixed modes for each radial order, such as in Mosser et al. (2012; see their Figure 2).

A first comparison between the predictions of our models (using Equation (1)) and the values of $\langle\Delta P\rangle_{\mathrm{a}}$ derived by Mosser et al. (2012) for a sample of Kepler giants is shown in Figure 7. All these models were computed without extra-mixing during the MS and during the He-B phases. It is clear that for the clump stars (low-mass stars with $\Delta v \sim 4 \mu \mathrm{Hz}$ ), these theoretical models systematically underestimate $\langle\Delta P\rangle_{\mathrm{a}}$ by $\sim 20 \%$.

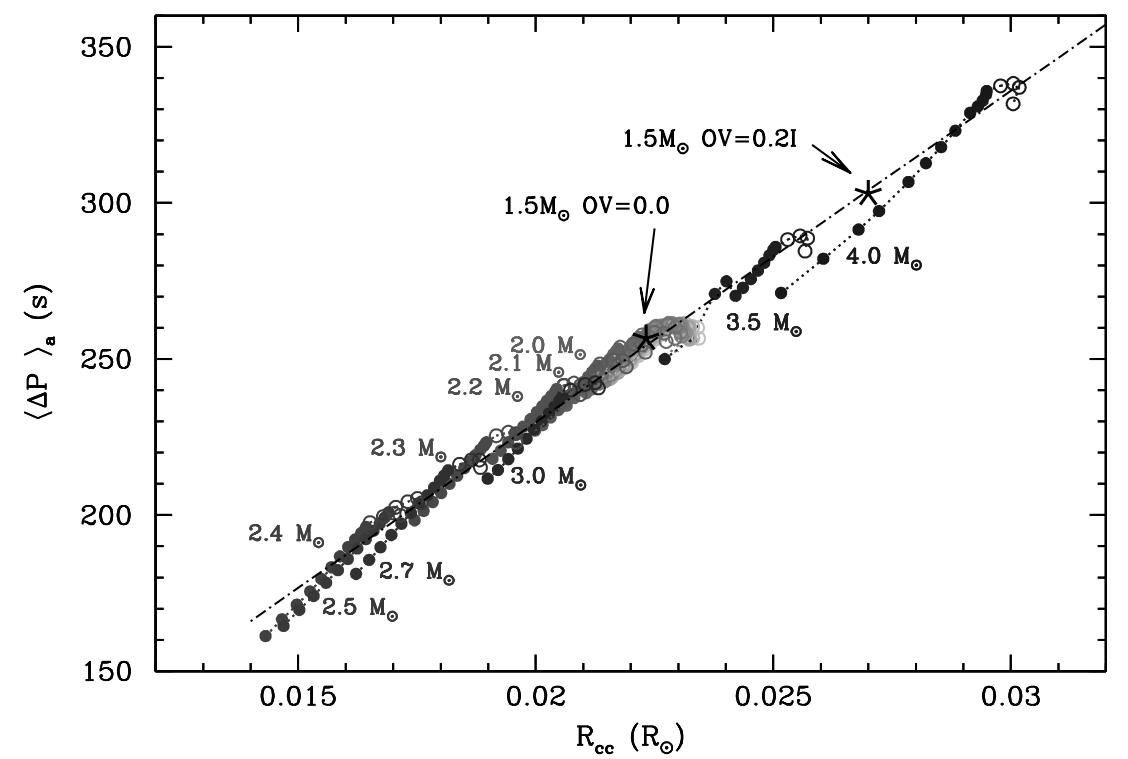

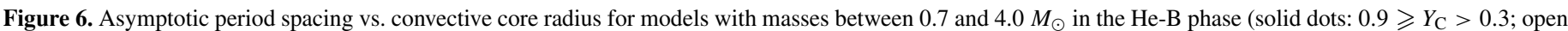

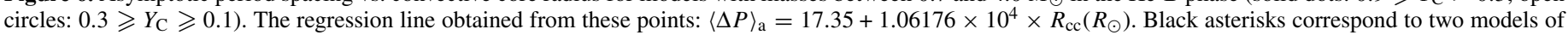
$1.5 M_{\odot}$ at $Y_{\mathrm{C}}=0.5$, without and with instantaneous and adiabatic overshooting during the He-B phase. 


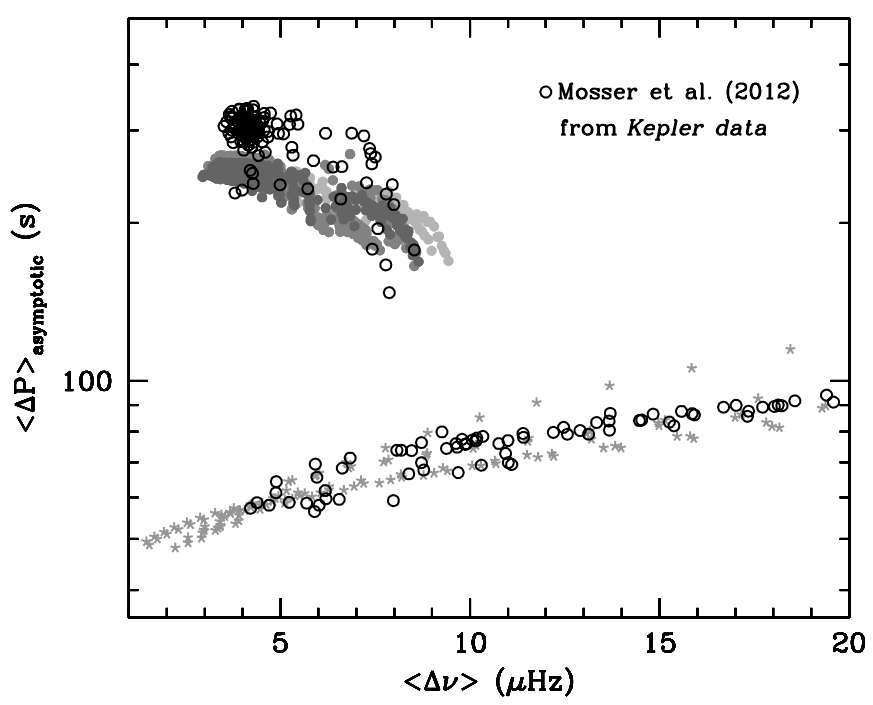

Figure 7. Theoretical asymptotic period spacing (Equation (1)) vs. average large frequency separation of radial modes for models on the RGB (asterisks) with masses $0.9,1.0,1.5,1.6,1.7$, and $2.0 M_{\odot}$ and for He-B models with masses between 0.7 and $3.0 M_{\odot}$ and three different chemical compositions $(Z, Y)$ : $(0.02,0.278)$ dark-gray dots, $(0.01,0.278)$ middle-gray dots, and $(0.02,0.25)$ light-gray dots. Black open circles are the asymptotic period spacing inferred by Mosser et al. (2012) for a sample of red giants in the Kepler field.

Mixing processes during the MS phase do not affect the central regions of low-mass stars in the He-B phase since the mass and the physical properties of the helium core are fixed by the onset of $\mathrm{He}$ burning in degenerate conditions. To increase $\langle\Delta P\rangle_{\mathrm{a}}$, we should decrease the contribution of $N$ in the innermost regions of the star, which can be easily done by extending the adiabatically stratified and chemically homogeneous core. We computed stellar models and oscillation frequencies for $M=1.5 M_{\odot}$, with central extra-mixing during the He-B evolution described by an instantaneous and adiabatic overshooting of length $\Lambda=0.2 H_{p}$. As expected, both the asymptotic and the minimum values of period spacing increase as the size of the homogeneous core increases. In Figure 8, we plot mode inertia and period spacing versus frequency for two models, computed with and without overshooting, of $1.5 M_{\odot}$ at $Y_{\mathrm{C}}=0.5$, and in Figure 6 we highlight with asterisks the values of $\langle\Delta P\rangle_{\mathrm{a}}$ for these models: without overshooting $\langle\Delta P\rangle_{\mathrm{a}}=255 \mathrm{~s}$, and with overshooting $\langle\Delta P\rangle_{\mathrm{a}}=305 \mathrm{~s}$. Note that the last values $\left(\langle\Delta P\rangle_{\mathrm{a}}\right.$ and $\left.R_{\mathrm{cc}}\right)$ are similar to those of a $4.0 M_{\odot}$ without overshooting. The exact $\langle\Delta P\rangle_{\mathrm{a}}$ value depends on the extension of the extra-mixed region, but also on the details of the mechanism at the origin of different $N$ profiles: a mechanical overshooting with an adiabatic temperature gradient in the extramixed region or an induced overshooting with a semi-convective layer outside the convective core. The latter will provide, for the same extension of the adiabatic region, a smaller period spacing than the former. The behavior shown in Figure 6 for $Z=0.02$ is also found for different metallicities. The only difference is the minimum values of $\langle\Delta P\rangle_{\mathrm{a}}$ and $R_{\mathrm{cc}}$ that depend slightly on $Z$. We found similar results using the MESA code (Paxton et al. 2011), considering models with and without overshooting during the He-B phase.

\section{CONCLUSIONS}

From the computation of stellar models with masses from 0.7 to $4.0 M_{\odot}$ and from the study of their adiabatic oscillation spectra, we find good agreement between the theoretical and observational behavior of period spacing. We also show the potential of dipole-mode period spacing in RGs to constrain the extension of central extra-mixing during the $\mathrm{H}$ - and $\mathrm{He}$-central burning phases:

1. The "observed" period spacing of stars with masses close to the transition value $\left(1.8-2.4 M_{\odot}\right.$ depending on chemical composition and mixing) presents an almost linear relation with the He-core mass, and therefore contains information on the extension of the convective core during the H-MS. These stars define the so-called secondary clump, and the value of their mass has been identified as the best index of the mixing processes during the MS in stellar clusters (Girardi 1999). Currently, seismology allows us to derive mass and secondary clump membership for a huge number of field RGs. Nevertheless, a fruitful exploitation of the enormous potential of seismic properties, allowing the comparison of observational results with predictions from synthetic stellar populations models, would also require information on chemical composition. So, a theoretical $\langle\Delta P\rangle_{\text {th-obs }}$ as a function of the total mass, initial chemical composition $(Z, Y)$, and evolutionary state $\left(Y_{\mathrm{C}}\right.$ or $\left.M_{\mathrm{He}}\right)$ will provide the expected $\langle\Delta P\rangle_{\text {th-obs }}$ distribution for synthetic stellar populations. Its comparison with observed distributions will shed some light on the mixing processes during the H-MS. It is also worth recalling that stars in the secondary clump spend a long time in the He-B phase, and that

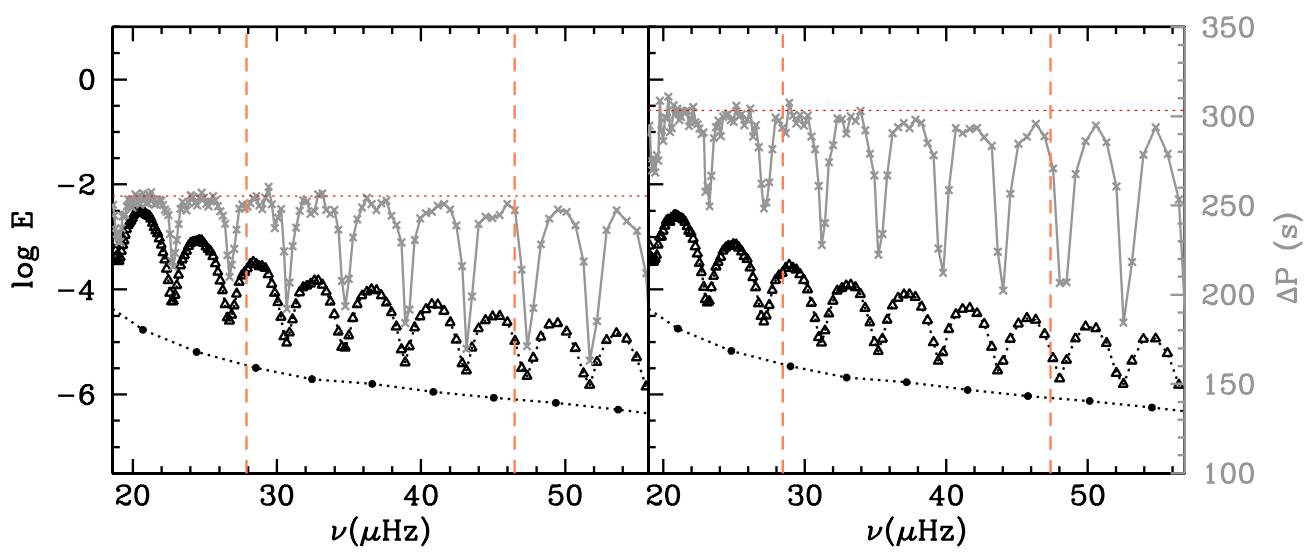

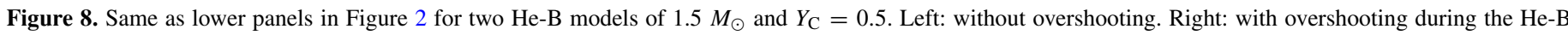
phase.

(A color version of this figure is available in the online journal.) 
their ages depend mainly on the MS extra-mixing (38\% larger for stars with MS overshooting) but are chemical composition independent (Sweigart et al. 1990).

2. The seismic properties of stars in the RC seem independent of core extra-mixing during MS, as low-mass stars reach the same kind of structure at He ignition. We have shown, however, that a linear relation exists between the asymptotic period spacing of dipole modes and the extension of the convective core in the He-B phase. Non-extra-mixing HeB models predict $\langle\Delta P\rangle_{a} \sim 20 \%$ smaller than values inferred from observations. If $\langle\Delta P\rangle_{\mathrm{a}}$ can be reliably derived from oscillation spectra, as suggested by Mosser et al. (2011) and Christensen-Dalsgaard (2012), then the basic seismic properties of oscillation spectra for RC stars can be used to constrain the extension and properties of core extra-mixing during the He-B phase.

When individual frequencies of dipole mixed modes will be available, a more direct comparison between models and observations shall be carried out, and will provide information not only on the extension of the central mixed region, but also on the detailed properties of the chemical composition gradient inside the star.

\section{REFERENCES}

Aerts, C., Thoul, A., Daszyńska, J., et al. 2003, Sci, 300, 1926 Andersen, J., Clausen, J. V., \& Nordstrom, B. 1990, ApJL, 363, L33 Bedding, T. R., Mosser, B., Huber, D., et al. 2011, Natur, 471, 608
Castellani, V., Degl'Innocenti, S., Girardi, L., et al. 2000, A\&A, 354, 150

Chiosi, C. 2007, in IAU Symp. 239, Convection in Astrophysics, ed. F. Kupka, I. Roxburgh, \& K. L. Chan (Prague, Czech Republic: IAU), 235

Christensen-Dalsgaard, J. 2004, SoPh, 220, 137

Christensen-Dalsgaard, J. 2012, in ASP Conf. Ser. 462, Progress in Solar/Stellar Physics with Helio- and Asteroseismology, ed. H. Shibahashi, M. Takata, \& A. E. Lynas-Gray (San Francisco, CA: ASP), 503

Degroote, P., Aerts, C., Baglin, A., et al. 2010, Natur, 464, 259

Deheuvels, S., \& Michel, E. 2011, A\&A, 535, A91

Di Mauro, M. P., Christensen-Dalsgaard, J., Kjeldsen, H., Bedding, T. R., \& Paternò, L. 2003, A\&A, 404, 341

Dupret, M.-A., Belkacem, K., Samadi, R., et al. 2009, A\&A, 506, 57

Dziembowski, W. A., \& Pamyatnykh, A. A. 2008, MNRAS, 385, 2061

Girardi, L. 1999, MNRAS, 308, 818

Girardi, L., Groenewegen, M. A. T., Weiss, A., \& Salaris, M. 1998, MNRAS, 301, 149

Grosjean, M., Dupret, M. A., Belkacem, K., et al. 2013, arXiv:1301.3249

Kippenhahn, R., \& Weigert, A. 1990, Stellar Structure and Evolution (Berlin: Springer)

Maeder, A., \& Mermilliod, J. C. 1981, A\&A, 93, 136

Miglio, A., \& Montalbán, J. 2005, A\&A, 441, 615

Montalbán, J., Miglio, A., Noels, A., Scuflaire, R., \& Ventura, P. 2010, ApJL, 721, L182

Mosser, B., Barban, C., Montalbán, J., et al. 2011, A\&A, 532, A86

Mosser, B., Goupil, M. J., Belkacem, K., et al. 2012, A\&A, 540, A143

Noels, A., Montalban, J., Miglio, A., Godart, M., \& Ventura, P. 2010, Ap\&SS, 328,227

Paxton, B., Bildsten, L., Dotter, A., et al. 2011, ApJS, 192, 3

Ribas, I., Jordi, C., \& Giménez, Á. 2000, MNRAS, 318, L55

Scuflaire, R., Montalbán, J., Théado, S., et al. 2008, Ap\&SS, 316, 149

Straniero, O., Domínguez, I., Imbriani, G., \& Piersanti, L. 2003, ApJ, 583, 878

Sweigart, A. V., Greggio, L., \& Renzini, A. 1990, ApJ, 364, 527

Tassoul, M. 1980, ApJS, 43, 469

Ventura, P., D’Antona, F., \& Mazzitelli, I. 2008, Ap\&SS, 316, 93

Ventura, P., Zeppieri, A., Mazzitelli, I., \& D’Antona, F. 1998, A\&A, 334, 953 